\title{
Localization based Ms-Mac Protocol to Enhance the Energy Efficiency in Sensor Networks
}

\author{
Jothi Kanna D. \\ Research Scholar \\ Department of computer science \\ PSGR Krishnammal college for \\ Women Coimbatore
}

\author{
Sophia Reena G. \\ Head of the Department \\ Department of Information Technology \\ PSGR Krishnammal college for \\ Women Coimbatore
}

\begin{abstract}
Wireless sensor networks (WSNs) are one of the most interesting research areas and have become very popular technology. Each and every node in the wireless sensor networks can be stationary or mobility depending on the application requirement. Optimizing energy in mobile sensor network is a current challenge in recent research. It mainly focuses to minimize the energy consumption, to reduce the delay and to improve the throughput while the sensor nodes are in movement. This paper proposes a new adaptive Localization Mobility-aware MAC protocol for Sensor networks (LMS-MAC) based on localization algorithm. The adaptation algorithms are used to localize mobile nodes and predict the quality of link it established at the link layer and reduces some level of energy consumption while the sensor nodes are in movement.
\end{abstract}

\section{General Terms}

Sensor Nodes, Localization, Distance estimation algorithm.

\section{Keywords}

Wireless sensor network, MS-MAC protocol, LMS-MAC localization techniques.

\section{INTRODUCTION}

A Wireless Sensor Network is a self-configuring network of small sensor nodes communicating among themselves using radio signals, and deployed in quantity to sense, monitor and understand the physical world. Wireless Sensor nodes are called motes. WSN provide a bridge between the real physical and virtual worlds. Wireless Sensor Networks (WSNs) can be defined as a self-configured and infrastructure-less wireless networks to monitor physical or environmental conditions, such as temperature, sound, vibration, pressure, motion or pollutants and to cooperatively pass their data through the network to a main location or sink where the data can be observed and analyzed. A sink or base station acts like an interface between users and the network. One can retrieve required information from the network by injecting queries and gathering results from the sink. Typically a wireless sensor network contains hundreds of thousands of sensor nodes. The sensor nodes can communicate among themselves using radio signals. A wireless sensor node is equipped with sensing and computing devices, radio transceivers and power components. To reduce the energy consumption in sensor nodes is a big challenge.

LMS-MAC protocol which is referred as localization based MAC protocol is proposed to reduce the energy consumption in sensor nodes. Number of MAC protocols proposed to save the energy in sensor nodes. When the sensor nodes are stationary it can consume some level of energy whereas it can consume high level of energy when the sensor nodes are in mobility. To overcome this issue in wireless sensor nodes the new adaptive LMS-MAC protocol is introduced. The proposed work is simulated using NS-2 software. The performance result is calculated by evaluating performance metrics such average energy consumption, end to end delay and Throughput.

\subsection{Architecture of WSN}

A WSN is a collection of sensor nodes which are deployed in sensor fields, which can collect and route the data back to the Base Station. A sensor node can be divided into four basic parts, the sensing unit, a processing unit, a transceiver unit, and a power unit. Localization is the heart of the routing principle in WSN. The position estimation system helps the sensor node to discover its position in the sensing field. The power unit gives the constant power supply to the sensor nodes.

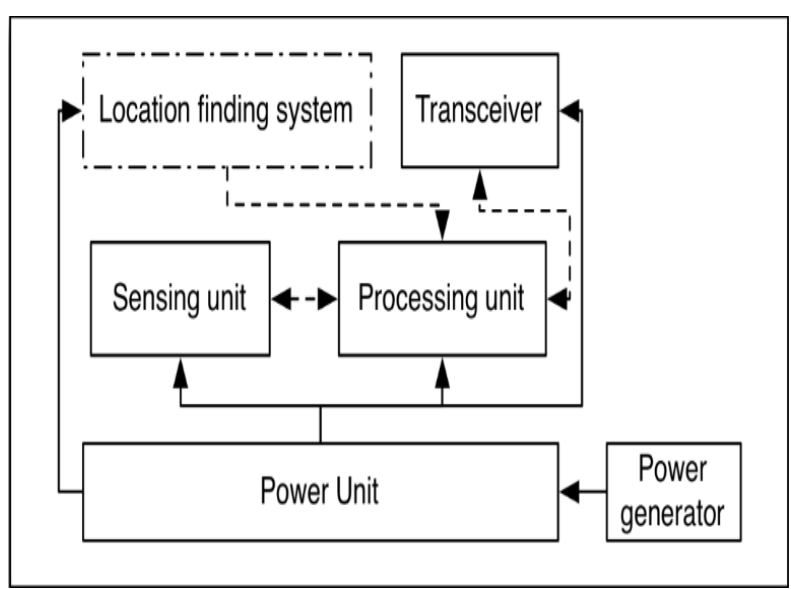

Fig. 1.WSN node model

The battery which is deployed in sensor nodes is not chargeable or changeable. Thus the life time of the sensor node is highly dependent on the battery lifetime.

\subsection{Types of wireless sensor networks}

Terrestrial WSNs consists of hundreds or thousands of WSN. These nodes can be deployed in any manner i.e. structured or an unstructured. If the nodes are deployed in an unstructured way, they are kept within the target area.

Underground WSNs are more costly when compared with other network. The equipment's which are used in these networks are expensive and to be maintained properly. These are effective to monitor the underground conditions. Problems faced are recharging the batteries and the loss of signal.

Underwater WSNs system comprises of sensor nodes which are deployed under the water. To collect the data from the 
underwater sensor nodes, vehicles are to be used. Propagation delay and sensor failure are a big challenge in underwater sensor network.

Multimedia WSNs sensor nodes can collect the information in the form of audio, video and imagining. These sensor nodes can be connected with cameras and microphones, which help in tracking and monitoring of the events. The visual display of the events is also maintained.

Mobile WSNs can move from one place to another. The main advantage is that they can provide coverage and superior

Schannel capacity. These mobile WSNs are able to adapt to the other static sensor systems.

\section{OBJECTIVE}

The main objective of the research is to minimize the energy consumption, to reduce the delay and to improve the throughput. Localization (LMS-MAC) based distance estimation method is used to find the distance between the head node and the other node. It is highly efficient to calculate its distance in order to decrease the usage of batteries. It ensures a successful and enhanced energy saving in sensor nodes.

\section{LITERATURE SURVEY}

Pratik S. Patel [1] proposed a Localization Algorithm for Mobile Sensor Nodes Using 3D Space in Wireless Sensor Network. This approach defines the range-based 3D localization algorithm that is accurate, anchor-free, scalable and physical position available. A novel combination of distance and direction measurement techniques introduced to estimate ranges between neighbors. Based on this information local coordinate systems are constructed and then converged to form a global network wide coordinate system ,which finally leads to nodes' absolute positions. Simulation results have shown that the algorithm achieves good trade-off between localization percentage and precision.

Tao Huang [2] proposed A Practical Localization Algorithm Based on Wireless Sensor Networks. The main contribution is to achieve higher localization accuracy, extra hardware equipment's are utilized by most of the existing localization algorithms, which increase the cost and greatly limit the range of location-based applications. A method which can effectively meet different localization accuracy requirements of most indoor and outdoor location services in realistic applications. The algorithm is composed of two phases: partition phase, in which the target region is split into small grids and localization refinement phase in which a higher accuracy location can be generated by applying a trick algorithm. A realistic demo system using our algorithm has been developed to illustrate its feasibility and availability. The results show that algorithm can improve the localization accuracy.

Priti Narwal [3] proposed a position estimation using localization technique in wireless sensor networks. This work includes to determine the location and orientation of sensor nodes. Location information is useful for both network organization and for sensor data integrity. In many wireless sensor network applications sensor nodes are required to know their locations with high degree of precision such as forest fire detection etc. Localization method helps in assisting sensor nodes to determine their location in sensor network. A technique called Multidimensional scaling is proposed which computes the position of nodes which are in the communication range of each other. This MDS-data analysis technique find out the relative position of nodes with accuracy sufficient enough for most of the applications so as to solve the problem of recreation.

Shweta Singh [4] proposed a Localization Techniques in Wireless Sensor Networks. The main contribution is to determine the geographical placement (location) of sensor nodes. Localization in wireless sensor network is not only useful for location determination but also it is important to

provide the base of routing. It can also be useful for density control, tracking and other communication aspects, so it is very important that every node should reports its location information very accurately. As we know that GPS is very accurate in location determination but it is expensive in terms of cost and energy of nodes, so it is not useful in wireless sensor networks. There are so many localization techniques (algorithms) are proposed for wireless sensor networks. Accuracy of localization techniques is most important before implementing it. The main focus is on the localization error. Localization error is the difference between the actual location and the estimated location of the sensor node. This helps the network designers to find out which techniques/algorithms are suitable for their application.

JerilKuriakose [5] proposed Localization in Wireless Sensor Networks: A Survey. This work involves in Wireless Sensor Networks (WSNs) to identify the current location of the sensornodes. A WSN consist of thousands of nodes that make the installation of GPS on each sensor node expensive and moreover GPS may not provide exact localization results in an indoor environment. Manually configuring location reference on each sensor node is also not possible for dense network. This gives rise to a problem where the sensor nodes must identify its current location without using any special hardware like GPS and without the help of manual configuration. In this review the localization techniques used by wireless sensor nodes to identify their current location.

P.K Singh [6] proposed Node localization in wireless sensor networks. Node localization is required to report the origin of events, assist group querying of sensors, routing and to answer questions on the network coverage. So, one of the fundamental challenges in wireless sensor network is node localization. This provides an overview of different approach of node localization discovery in wireless sensor networks. The overview of the schemes proposed by different scholars for the improvement of localization in wireless sensor networks is also presented. Future research directions and challenges for improving node localization in wireless sensor networks are also discussed.

Aditi Shrivastava [7] proposed Localization Techniques for Wireless Sensor Networks. In this work it involves in Wireless sensor networks (WSNs) that have recently emerges as promising technology in wireless communication field and gained special attention by research groups. It uses small and cheap gadgets with low energy requirements and limited on board computing resources which communicates with each other's or base stations without any pre-defined infrastructure. The property of being infrastructure less makes it suitable in distinctive application situations including remote monitoring, disaster management, military applications and biomedical health observing devices. In many of these applications, node localization is unavoidably one of the important system parameters for example in target tracking if the nodes are not able to obtain the accurate location information, the related task cannot be performed. It is also helpful in routing, network coverage and quarry management of sensors. In general the 
localization techniques are ordered into two general classifications: range based and range free. The discussion on various localization algorithms with their applicable areas, requirements and limitations. Moreover, on conclusion it compares these localization algorithms and analyzes the future research directions for the localization algorithms in WSNs.

Anouar Abdelhakim Boudhir [8] proposed New Technique of Wireless Sensor Networks Localization based on Energy Consumption. The main contribution is to improve the quality of service and communication. These provides some techniques of localization techniques on wireless sensor networks and propose a localization method based on the angle of transmission of directional antenna and the energy consumption of the node when sending a data. Then we compare the mentioned method to TDOA technique (Time Differential Of Arrival) applied on the context of mobility characterizing the transport domain and compare their performance when localizing sensors in this kind of applications.

Pratik Biswas [9] Semidefinite Programming Approaches for Sensor Network Localization with Noisy Distance Measurements. The main contribution is to determine the positions of the sensor nodes in a network given incomplete and inaccurate pairwise distance measurements. Such distance data may be acquired by a sensor node by communicating with its neighbors. semidefinite programming (SDP) based approach for solving the graph realization problem, of which the sensor network localization problems is a special case. Error bounds are derived from the SDP formulation. The sources of estimation error in the SDP formulation are identified. The SDP solution usually has a rank higher than the underlying physical space, which when projected onto the lower dimensional space generally results in high estimation error. The two improvements to ameliorate such a difficulty. First, a regularization term in the objective function that can help to reduce the rank of the SDP solution. Second, the points estimated from the SDP solution as the initial iterate for a gradient-descent method to further refine the estimated points. A lower bound obtained from the optimal SDP objective value can be used to check the solution quality. Experimental results are presented to validate our methods and show that they outperform existing SDP methods.

As a result, many researchers proposed a techniques and algorithms for improving energy efficiency in wireless sensor networks. None of them deals with Localization based MSMAC protocol to enhance the energy efficiency in sensor networks. The localization has been handled in different ways. In our proposed work localization technique is used based on the MAC protocols which increase high level of energy in sensor nodes.

\section{METHODOLOGIES}

In general Wireless Sensor Networks (WSNs) use batteries for energy supply and these batteries are irreplaceable as well as non-chargeable. So that energy efficient communication is vital for increasing the life time of the network. Many research papers discussed this issue as well as develop many routing protocol to overcome this problem. However, any node failure due to battery depletion will lead to loss of data in single path, in such case a new algorithm have been proposed to save some level of energy. This research paper proposes a new adaptive Localization Mobility-aware MAC protocol for Sensor networks (LMS-MAC) based on localization algorithm. The adaptation algorithms are used to localize mobile nodes and predict the quality of link it established at the link layer and reduces some level of energy consumption while the sensor nodes are in movement. This research is trying to evaluate the distance estimation method. The simulation results show that proposed LMS-MAC can achieve significantly better energy and better throughput seamlessly with little or even none redundancy. This paper presents a new adaptive localization mobility-aware MAC protocol for sensor networks (LMS-MAC). By doing this way, the new mobility-aware LMS- MAC protocol can work very energy-efficiently when the network is mobility, whereas it can maintain much level of network performance with the mobile sensors. Node localization is commonly employed in wireless networks. For example, it is used to improve routing and enhance energy.

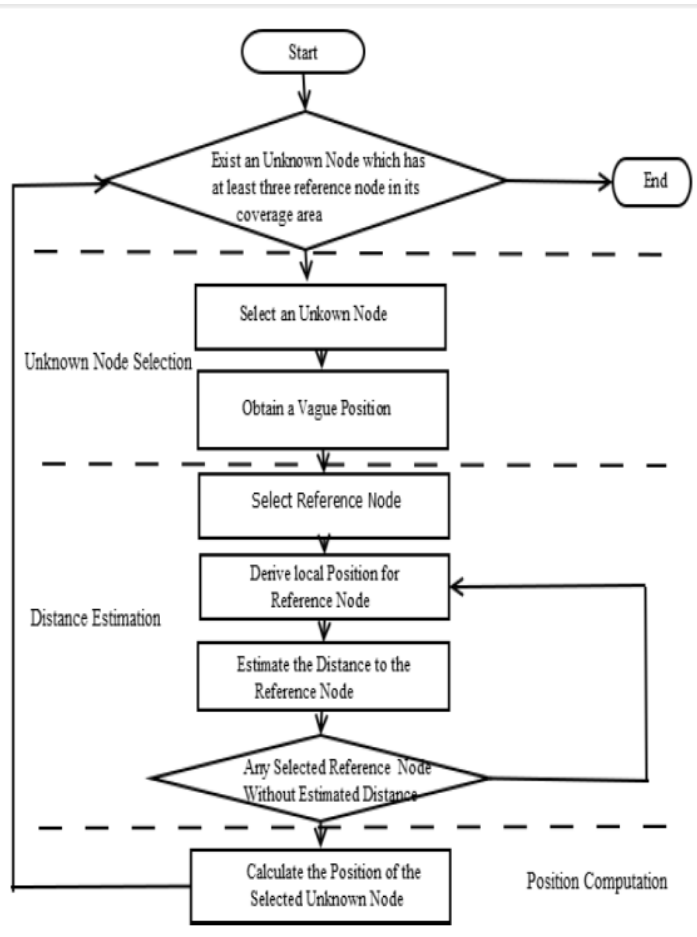

Fig.2 Frame work of localization technique in WSNs

\section{ALGORITHM USED}

Localization algorithms can be classified as range-free or range-based. Range-based algorithms use location metrics such as AoA, ToA, RSS, and to estimate the distance between two nodes. Proximity sensing between nodes is typically the basis for range-free algorithms. A tradeoff exists since rangebased algorithms are more accurate but also more complexes. However, in applications such as target tracking, localization accuracy is very important. Range based localization algorithms mainly focus on estimating the distance and angle between the sensor nodes. These algorithms first calculate the distance between nodes and with the help of geometrical principles they compute the location for the same. These algorithms use sophisticated hardware to find out the range metrics such as AOA (Angle of arrival), TOA (Time of arrival), and RSSI (received signal strength indication). There should be communication between localized and un-localized node in its vicinity for determining the geometrical placement or position (location) of un-localized node.

\subsection{Angle of Arrival (AOA)}

This scheme can be used to find the location of the unlocalized node. It can be determined by estimating absolute 
or relative angles between the neighbor's. It is also called as direction of arrival (DOA). It is measured in degree with clockwise direction from the north.
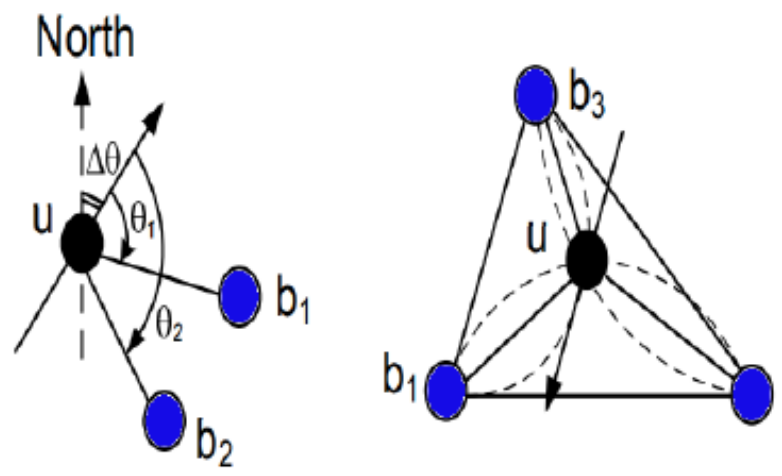

Fig. 3 Angle of arrival

\subsection{Time of Arrival (TOA)}

This scheme is used to find the location of the unlocalized node by calculating the speed of wavelength and time of radio signals transverses between anchor node and unlocalized node. All the sensor nodes will transmit the signal to the neighbour node with the same velocity. After receiving the signal will be send back to the transmitter.

\subsection{Received Signal Strength Indicator (RSSI)}

Radio signal energy is an electromagnetic wave. These wave strength decreases when it starts moving forward. When transmitting the data packet by sensor nodes the RSSI values is calculated. The sensor node will hear the transmitted signal by the access point. The absolute measurement cannot be shown by RSSI. By using this, the estimation of distance between the transmitter and the receiver is calculated.

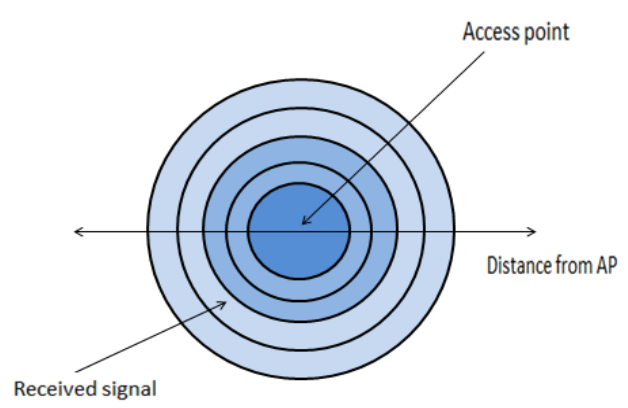

Fig.4 Received signal strength and separation distance between access points and receivers

\section{EXPERIMENTAL RESULTS}

The proposed system is evaluated in Network Simulator 2 tool as NS2.34 version and we have used around 40 nodes starts from 0-39. The energy efficiency is enhanced by localization based algorithms. The algorithm called distance estimation is used to calculate the angle between the sensor nodes. The parameters like average energy consumption, throughput and end to end delay are evaluated for comparison of the existing and the proposed system.

\subsection{Average energy consumption}

It measures the average difference between the initial level of energy and the final level of energy that is left in each node. Energy consumption of the node is the subsequent metric to be conducted. LMS-MAC energy consumption is much lesser when compared to other MS-MAC localization scheme. It keeps node energy consumption of node at reduced state through increment of network size normally occurs. The energy efficiency of LMS-MAC is more consistent as the results shows from the experiment conducted and the effect of increasing the size of the network shows smaller effects and comparing it to other localization schemes and their performances diminishes when the network size becomes bigger.

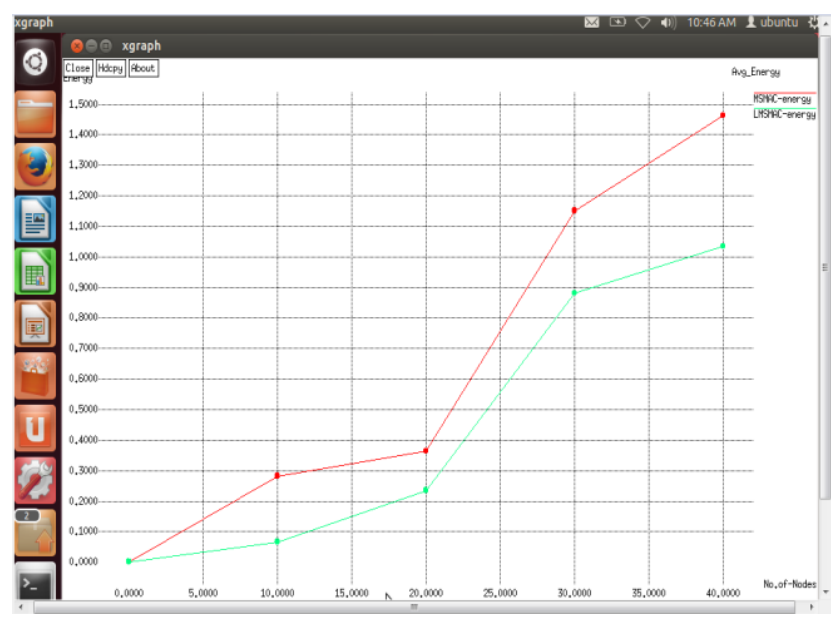

Fig.5 Average energy consumption

\subsection{Throughput evaluation}

Throughput is defined as the total number of packets delivered over the total simulation time. Mathematically, it can be defined as:

$$
\text { Throughput }=\mathrm{N} / 1000
$$

Where $\mathrm{N}$ is the number of bits received successfully by all destination.

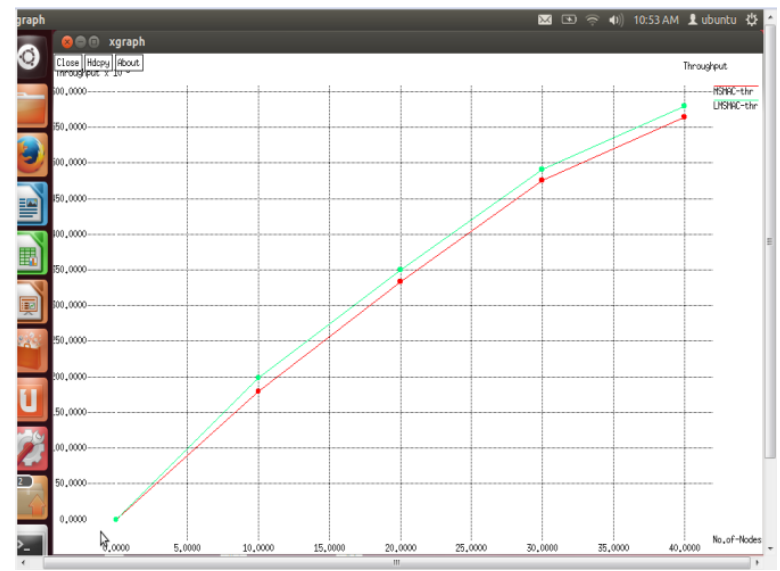

Fig.6 Throughput evaluation

Fig.6 shows the throughput comparison of the proposed LMSMAC protocol approach and the existing MS-MAC. It is noted that the proposed LMS-MAC protocol attains higher throughput when compared with the existing MS-MAC. The reason is that, the probability of the processing time decreases with localization method. 


\subsection{End to End delay}

It defines as the average time taken by a data packet to arrive at the destination. Only the data packets that successfully delivered to destinations that counted. The average packet delay is described. Showing that in comparison with other schemes the LMS-MAC having the shortest delay is a great benefit to the scheme. Through the use of the LMS-MAC, as estimated the data packets can easily reach the destination with shortest time.

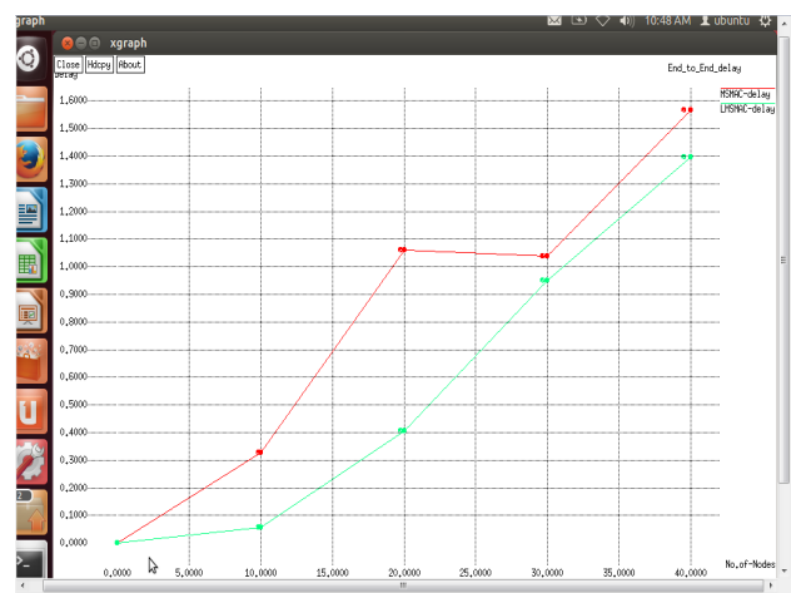

Fig.7 End to End delay

Our proposed system decreases the energy consumption by using localization technique. Thus the energy consumed by each node is decreased.

\section{CONCLUSION}

This paper proposes the Localization Mobility-aware MAC protocol for Sensor networks (LMS-MAC) based on localization algorithm. The adaptation algorithms are used to localize mobile nodes and predict the quality of link it established at the link layer and reduces some level of energy consumption while the sensor nodes are in movement. It has been shown through both theoretical analysis and simulation results that to minimize the energy consumption, to reduce the delay and to improve the throughput while the sensor nodes are in movement.

The LMS-MAC protocol identifies the location of the node and it is controlled, based on the presence of mobile nodes and speed. It also creates a new connection before it loses all the connection it provides better QoS performance. As a future work, our attention is to deeply evaluate and analyze the performance of proposed protocol by studying other performance metrics such as other localization techniques. Further, we will study the impacts of the energy consumption and the throughput evaluation on the performance metrics.

\section{REFERENCES}

[1] Pratik S. patel, Jignesh R.patel, "Localization Algorithm for Mobile Sensor Nodes Using 3D Space in Wireless Sensor Network" , International Journal on Recent and Innovation Trends in Computing and Communication, Volume: 2, Issue: 1, pp. (58 - 62 ) January 2014 . ISSN: 2321-8169

[2] Tao Huang, Zhikui Chen, Feng Xia, Cheng Jin, Liang Li, "A Practical Localization Algorithm Based on Wireless Sensor Networks".
[3] Priti Narwal1, Dr. S.S. Tyagi , "Position estimation using localization technique in wireless sensor networks", International Journal of Application or Innovation in Engineering \& Management (IJAIEM), Volume 2, Issue 6, June 2013. ISSN $2319-4847$

[4] Shweta Singh, Ravi Shakya, Yaduvir Singh, "Localization Techniques in Wireless SensorNetworks", International Journal of Computer Science and Information Technologies, Vol. 6 (1), 2015, pp (844-850). ISSN: 0975-9646

[5] JerilKuriakose, Sandeep Joshi , V.I. George, "Localization in Wireless Sensor Networks: A Survey" , CSIR Sponsored X Control Instrumentation System Conference - CISCON-2013.

[6] P.K Singh, Bharat Tripathi, Narendra Pal Singh, "Node localization in wireless sensor networks", International Journal of Computer Science and Information Technologies, Vol. 2 (6), 2011,PP.( 2568-2572). ISSN: 0975-9646

[7] Aditi Shrivastava, Priya Bharti, "Localization Techniques for Wireless Sensor Networks",

International Journal of Computer Applications, PP. (0975 8887) Volume 116 - No. 12, April 2015.

[8] Anouar Abdelhakim Boudhir, Bouhorma Mohamed, Ben Ahmed Mohamed, "New Technique of Wireless Sensor Networks Localization based on Energy Consumption", International Journal of Computer Applications, PP(0975 - 8887), Volume 9- No.12, November 2010.

[9] Pratik Biswas, Tzu-Chen Liang, Kim-Chuan Toh, TaChung Wang and Yinyu Ye, "Semidefinite Programming Approaches for Sensor Network Localization with Noisy Distance Measurements".

[10] S. Gezici, Z. Tian, G. B. Giannakis, H. Kobayashi, A. F. Molisch, H. V. Poor, and Z. Sahinoglu, "Localization via ultra-wideband radios: a look at positioning aspects for future sensor networks", IEEE Signal Processing Magazine, vol. 22, no. 4, pp. 70\{84, Jul. 2005.

[11] R. Huang and G. V. Zaruba, "Beacon deployment for sensor network localization", inIEEE Wireless Communications and Networking Conference, Mar. 2007, pp. $3188\{3193$.

[12] N. Bulusu, J. Heidemann, and D. Estrin, "GPS-less lowcost outdoor localization forvery small devices," IEEE Personal Communications, vol. 7, no. 5, pp. $28\{34$ Oct. 2000

[13] H. Wymeersch, J. Lien, and M. Z. Win, "Cooperative localization in wireless networks,"Proc. IEEE, vol. 97, no. 2, pp. $427\{450$, Feb. 2009

[14] X. Wang, Z.Wang, and B. O'Dea, "A TOA-based location algorithm reducing the errors due to non-line-ofsight (NLOS) propagation," IEEE Transactions on Vehicular Technology, vol. 52, no. 1, pp. 112\{116, Jan. 2003.

[15] K. C. Ho and M. Sun, "Passive source localization using time differences of arrival and gain ratios of arrival," IEEE Transactions on Signal Processing, vol. 56, no. 2, pp. $464\{477$, Feb. 2008. 